\title{
Jameel's Two and Three-Dimensional Stressed Closed Form Models are Indeed IFRS 9 Complaint Models
}

\author{
Jamilu Auwalu Adamu ${ }^{1}$ \\ ${ }^{1} 118$ National Mathematical Centre, Nigeria \\ Correspondence: Jamilu Auwalu Adamu, 118 National Mathematical Centre, Abuja, Nigeria. Tel: 234-8038-679- \\ 094. E-mail: whitehorseconsult@yahoo.com
}

Received: June 3, 2018; Accepted: June 16, 2018; Published: June 24, 2018

\begin{abstract}
In the paper entitled "IFRS 9 Measurement of Financial Instruments 2018: Jameel's Non-Normal Brownian Motion Models are Indeed IFRS 9 Complaint Models", the author was able to incorporated a forward-looking information $\left\{W_{J B(t)}\right\}$ satisfied Jameel's Criterion and Geometric average of only positive Economic forecasts of the future Macroeconomic parameters $\left\{\left(\mu_{A}\right)\right.$ and $\left.\left(\sigma_{A}\right)\right\}$ using Jameel's Contractional-Expansional Stress Methods and Jameel's substitutions $\left\{\left(\mu_{A} \pm \sigma_{A} W_{J B}(t)\right)\right\}, \mu_{A}$ is POSITIVE INFINITESIMAL, $\sigma_{A} \geq 1$ into Geometric Brownian Motion, Biagin, Cox-Ingersoll-Ross, Ornstein-Uhlenbeck process, Vasicek, Black-Karasinki, Chen, Kalotay-Williams-Fabozzi, Longstaff-Schwatz, Ho-Lee, Hull and White, and Black-Derman-Toy Models for Pricing Stocks, Bitcoin, Indexes, ETFs, and Leveraged ETFs, Bonds, Interest Rate Movements, Caps, Floors, European Swaptions, and Bond Options. However, in this paper, the author has attempted to further stress the research models by REPLACING the forward-looking information $\left\{W_{J B(t)}\right\}$ with the LINEAR COMBINATIONS of the forward-looking information(s): (a) $\left\{\left(\mu_{A} \pm \sigma_{A} W_{J B 1}(t) \pm \sigma_{A} W_{J B 2}(t)\right)\right\}, \mu_{A}$ is POSITIVE INFINITESIMAL, $\sigma_{A} \geq 1, W_{J B 1}(t)$ is a LOG-LOGISTIC (3P) and $W_{J B 2}(t)$ is a CAUCHY; (b) $\left\{\left(\mu_{A} \pm \sigma_{A} W_{J B 1}(t) \pm \sigma_{A} W_{J B 2}(t) \pm \sigma_{A} W_{J B 3}(t)\right)\right\}, \mu_{A}$ is POSITIVE INFINITESIMAL, $\sigma_{A} \geq 1, W_{J B 1}(t)$ is a LOG-LOGISTIC (3P), $W_{J B 2}(t)$ is a CAUCHY and $W_{J B 3}(t)$ is a BURR (4P). The paper tested the performances of TWO and THREE-DIMENSIONALTYPES of the proposed Jameel's stressed closed form solutions with the aid of Chevron Corporation (CVX) Stock data extracted from yahoo finance, time series from $2014-1991$. The results were fascinatingly interesting, impressive, viable and reliable, sophisticated, and complaint with IFRS 9 since they incorporated MORE forward-looking information(s) and Economic forecasts of the future macroeconomic parameters thereby minimizing the differences between market prices and models prices.
\end{abstract}

Keywords: Forward-Looking Information, Macroeconomic Parameters, Log-Logistic (3P), Cauchy, Burr (4P)

\section{Introduction}

In this paper, the Author attempted to INCORPORATE forward-looking information : (a) $\left\{\left(\mu_{A} \pm \sigma_{A} W_{J B 1}(t) \pm\right.\right.$ $\left.\left.\sigma_{A} W_{J B 2}(t)\right)\right\}, \mu_{A}$ is POSITIVE INFINITESIMAL, $\sigma_{A} \geq 1, W_{J B 1}(t)$ is a LOG-LOGISTIC $(3 \mathrm{P})$ and $W_{J B 2}(t)$ is a CAUCHY; (b) $\left\{\left(\mu_{A} \pm \sigma_{A} W_{J B 1}(t) \pm \sigma_{A} W_{J B 2}(t) \pm \sigma_{A} W_{J B 3}(t)\right)\right\}, \mu_{A}$ is POSITIVE INFINITESIMAL, $\sigma_{A} \geq 1$, $W_{J B 1}(t)$ is a LOG-LOGISTIC (3P), $W_{J B 2}(t)$ is a CAUCHY and $W_{J B 3}(t)$ is a BURR (4P) where $W_{J B 1}(t)$, 
$W_{J B 2}(t)$ and $W_{J B 3}(t)$ satisfies Jameel's Criterion with $\left\{\left(\mu_{A}\right)\right.$ and $\left.\left(\sigma_{A}\right)\right\}$ are Geometric average of only positive Economic forecasts of the future Macroeconomic scenarios and define by Geometric Means of only positive Arithmetic Means of the Underlying Asset Return and Returns of the future economic forecasts of macroeconomic parameters and Geometric Volatility of only positive Arithmetic Means of the Underlying Asset Return and Returns of the future economic forecasts of macroeconomic parameters respectively. The paper apply Jameel's Contractional-Expansional Stress Methods and Jameel's substitutions to NON-NORMALLY stress the closed form solutions of Geometric Brownian Motion, Biagin, Cox-Ingersoll-Ross, Ornstein-Uhlenbeck process, Vasicek, Black-Karasinki, Chen, Kalotay-Williams-Fabozzi, Longstaff-Schwatz, Ho-Lee, Hull and White, and BlackDerman-Toy Models for Pricing Stocks, Bitcoin, Indexes, ETFs, and Leveraged ETFs, Bonds, Interest Rate Movements, Caps, Floors, European Swaptions, and Bond Options using (i) $\left(\mu_{A} \pm \sigma_{A} W_{J B 1}(t) \pm \sigma_{A} W_{J B 2}(t)\right)$, whenever $\sigma_{A}>1, \mu_{A}$ is positive infinitesimal; (ii) $\left(\mu_{A} \pm W_{J B 1}(t) \pm W_{J B 2}(t)\right)$, whenever $\sigma_{A}=1, \mu_{A}$ is positive infinitesimal; (iii) $\left( \pm \sigma_{A} W_{J B 1}(t) \pm \sigma_{A} W_{J B 2}(t)\right)$, whenever $\sigma_{A}>1, \mu_{A}=0$; and (iv) $\left( \pm W_{J B 1}(t) \pm\right.$ $\left.W_{J B 2}(t)\right)$, whenever $\sigma_{A}=1, \mu_{A}=0$ for TWO-DIMENSIONAL stressed closed form models and (1) $\left(\mu_{A} \pm \sigma_{A} W_{J B 1}(t) \pm \sigma_{A} W_{J B 2}(t) \pm \sigma_{A} W_{J B 3}(t)\right)$, whenever $\sigma_{A}>1, \mu_{A}$ is positive infinitesimal; (2) ( $\mu_{A} \pm$ $\left.W_{J B 1}(t) \pm W_{J B 2}(t) \pm W_{J B 3}(t)\right)$, whenever $\sigma_{A}=1, \mu_{A}$ is positive infinitesimal; (3) $\left( \pm \sigma_{A} W_{J B 1}(t) \pm\right.$ $\left.\sigma_{A} W_{J B 2}(t) \pm \sigma_{A} W_{J B 3}(t)\right)$, whenever $\sigma_{A}>1, \mu_{A}=0$; and (4) $\left( \pm W_{J B 1}(t) \pm W_{J B 2}(t) \pm W_{J B 3}(t)\right)$, whenever $\sigma_{A}=1, \mu_{A}=0$ for THREE-DIMENSIONAL stressed closed form models.

Finally, the paper round up with the test of performances of the proposed 2 and 3-dimentional stressed closed form models using Chevron Corporation (CVX) Stock data extracted from yahoo finance, time series from 2014 - 1991.

\section{Methods and Materials}

\subsection{Materials}

\subsubsection{Linear Combination}

In Mathematics, a Linear Combination is an expression constructed from a set of terms by multiplying each term by a constant and adding the results. A Linear Combination of $x$ and $y$ is given by $a x+b y$.

\subsubsection{Jameel's Criterion:}

Under this criterion, we run the goodness of fits test such that:

i. We accept if the Average of the ranks of Kolmogorov Smirnor,Anderson Darling and Chi-squared is less than or equal to Three (3)

ii. We must choose the Probability Distribution follows by the data ITSELF regardless of its Rankings

iii. If there is tie, we include both the Probability Distributions in the selection

iv. At least Two (2) Probability Distributions must be included in the selection

v. We select the most occur Probability Distribution as the qualify candidate in each case of test of goodness of fit.

vi. Criterion Enhancement Axiom: Thode (2012) intensively discussed about the Best Goodness of Fit Tests such as Kolmogorov Smirnov (KS) Test, Anderson-Darling Test, Jarque and Bera (JB) Test, Shapiro Wilk (SW) Test, Cramer-Von Mises Test, Pearson ( $\chi^{2}$ Godness of Fit $)$ Test, Lilliefors Corrected K-S Test, 
D’AgostinoSkewness Test, Anscombe-Glynn Kurtosis Test, D’Agostino-Pearson Omnibus Test. Let $\left\{T_{1}, T_{2}, \ldots, T_{n}\right\}$ be the set of such Best Goodness of Fit Tests, $\left\{x_{1}, x_{2}, \ldots, x_{n}\right\}$ be their RANKS respectively then the generality of (i) can be expressed (or enhanced) if $\frac{\left(x_{1}+x_{2}+\ldots+x_{n}\right)}{n} \leq a$, where $0<a \leq n, n \in N$ or equivalently, $x_{1}+x_{2}+\ldots+x_{n} \leq$ an

vii. Last Unit Axiom: let $W_{J B}(t)$ be such that it satisfied axioms (i) to (iv). Let $\left\{r_{1}, r_{2}, \ldots, r_{n}\right\}$ be the ranks of fitness test of $W_{J B}(t)$ obtained from the tests $\left\{T_{1}, T_{2}, \ldots, T_{n}\right\}$ respectively then if $\forall i \in\{1,2, \ldots, n\}, r_{i}=1$ regardless of the Time Series, Company and so on. Consequently, if for all fitness test runs, turn out to be the same $W_{J B}(t)$ then the PREDICTED PRICE PATH will finitely coincides many times with the REAL PRICE PATH of the stock under consideration.

\subsubsection{Top Fat-Tailed Probability Functions using Jameel's Criterion as of 2015}

Using Jameel's Criterion, Jamilu (2015) considered Eleven (11) out of Fifty (50) World's Biggest Public Companies by FORBES as of 2015 Ranking regardless of the platform in which they are listed, Number of the Research Companies, Time Series (Short or Long), Old or Recently listed Companies using the time series from 2014 - 2009 with the aim of finding the Best Fitted Fat - Tailed Stocks Probability Distributions. However, in this research paper, the Author considered Top Two (2) and 4th Stocks Fat-Tailed Probability Functions thereby comparing the performances of the Proposed Jameel's Stressed Closed Form Prices, Normal (Standard Brownian Motion) Prices with Market (Real) Prices as shown below:

Log - Logistic (3P) Probability Distribution $\left(1^{\text {st }}\right)$ :

$$
f(x ; \mu, \sigma, \xi)=\frac{\left(1+\frac{\xi(x-\mu)}{\sigma}\right)^{-(1 / \xi+1)}}{\left[1+\left(1+\frac{\xi(x-\mu)^{-1 / \xi}}{\sigma}\right)\right]^{2}} ; x \geq \mu
$$

Cauchy Probability Distribution $\left(2^{\text {nd }}\right)$ :

$$
f(x ; \mu, \sigma, \pi)=\left(\pi \sigma\left(1+\left(\frac{x-\mu}{\sigma}\right)^{2}\right)\right)^{-1} ;-\infty<x<+\infty
$$

Burr (4P) Probability Distribution $\left(4^{\text {th }}\right)$ :

$$
f(x ; \alpha, \beta, \gamma)=\frac{a k\left(\frac{x-\gamma}{\beta}\right)^{\alpha-1}}{\beta\left(1+\left(\frac{x-\gamma}{\beta}\right)^{\alpha}\right)^{k+1}} ; \alpha, \beta, k>0
$$

\subsection{Methods}

2.2.1 Geometric Brownian Motion Stock Pricing

The Brownian Motion $S(t)=\mu t+\sigma W(t), \mu \in R, \sigma \geq 0$, the Wiener Process $W(t)$ is indeed a Random 
GAUSSIAN (NORMAL) Function with mean zero and variance $t$ as shown by Norbert-Wiener in the early 1920s. Mathematically, $W(t)$ is a NORMALLY DISTRIBUTED random variable with expected value zero and variance $t$. Therefore it is true that $W(t) \sim N(0, t)$. From the fact that the process $\{W(t): t \geq 0\}$ is a GAUSSIAN (NORMAL) with mean zero and variance $t$ then $S(t)=\mu t+\sigma W(t)$ is a NORMAL BROWNIAN MOTION STOCK PRICE.

2.2.2 Propose 2-Dimentional Jameel's Stressed Stock Pricing Models for IFRS 9 Compliance

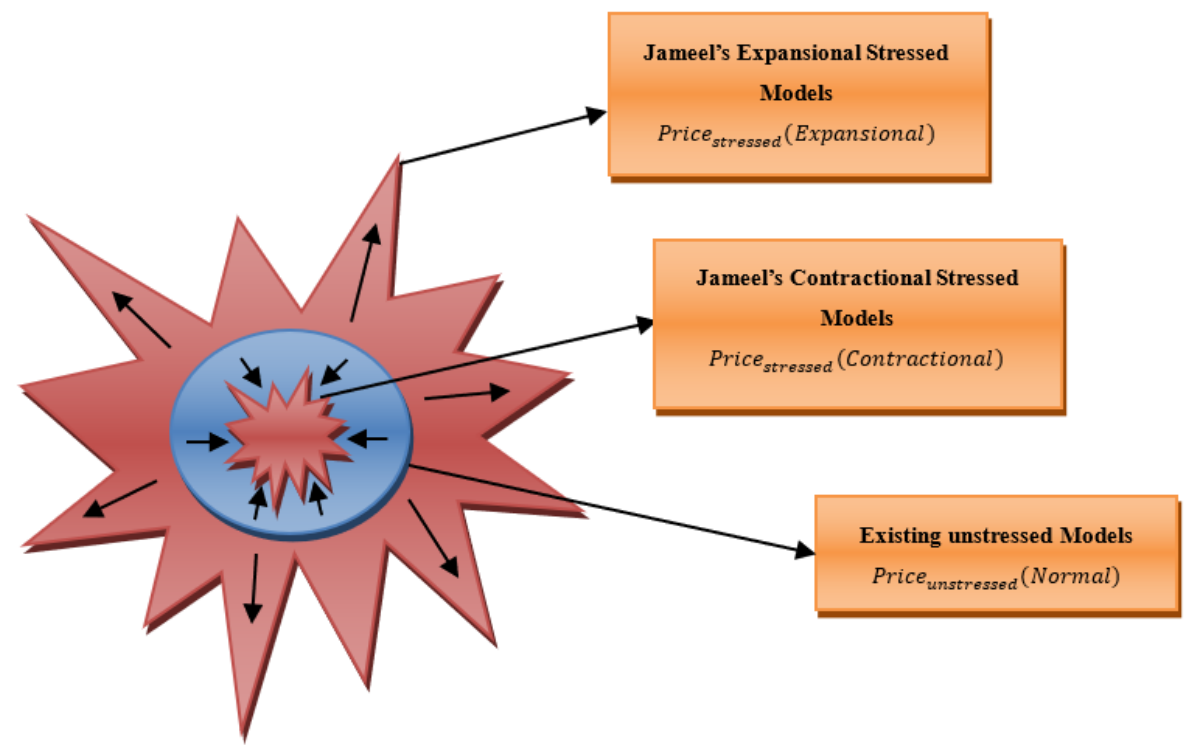

Figure 1. Jameel's Contractional-Expansional Stressed Methods

Applying Jameel's Criterion and Jameel's Contractional-Expansional Stressed Methods to REPLACE $\left\{W_{J B}\right\}_{t \geq 0}$ of the Geometric Brownian Motion with the JAMEEL'S SUBSTITUTIONS FOR IFRS 9 COMPLIANCE for

TWO-DIMENSIONAL stress closed form (i) $\left(\mu_{A} \pm \sigma_{A} W_{J B 1}(t) \pm \sigma_{A} W_{J B 2}(t)\right)$, whenever $\sigma_{A}>1$, $\mu_{A}$ is positive infinitesimal; (ii) $\left(\mu_{A} \pm W_{J B 1}(t) \pm W_{J B 2}(t)\right)$, whenever $\sigma_{A}=1, \mu_{A}$ is positive infinitesimal; (iii) $\left( \pm \sigma_{A} W_{J B 1}(t) \pm \sigma_{A} W_{J B 2}(t)\right)$, whenever $\sigma_{A}>1, \mu_{A}=0$; and (iv) $\left( \pm W_{J B 1}(t) \pm W_{J B 2}(t)\right)$, whenever $\sigma_{A}=1$, $\mu_{A}=0$, then we have the following Propose Jameel's Stressed Closed Form Stocks Pricing Models TYPES for IFRS 9 Compliance as:

TYPE 1:

$\left(S_{J B}(t)\right)_{\text {Stressed }}=p_{0} \exp \left(\mu t+\sigma\left(\mu_{A} \pm \sigma_{A} W_{J B 1}(t) \pm \sigma_{A} W_{J B 2}(t)\right)\right)$,

whenever $\sigma_{A}>1, \mu_{A}$ is positive infinitesimal;

TYPE 2:

$\left(S_{J B}(t)\right)_{\text {Stressed }}=p_{0} \exp \left(\mu t+\sigma\left(\mu_{A} \pm W_{J B 1}(t) \pm W_{J B 2}(t)\right)\right)$, 
whenever $\sigma_{A}=1, \mu_{A}$ is positive infinitesimal;

TYPE 3:

$\left(S_{J B}(t)\right)_{\text {Stressed }}=p_{0} \exp \left(\mu t+\sigma\left( \pm \sigma_{A} W_{J B 1}(t) \pm \sigma_{A} W_{J B 2}(t)\right)\right)$,

whenever $\sigma_{A}>1, \mu_{A}=0$;

TYPE 4:

$\left(S_{J B}(t)\right)_{\text {stressed }}=p_{0} \exp \left(\mu t+\sigma\left( \pm W_{J B 1}(t) \pm W_{J B 2}(t)\right)\right)$,

whenever $\sigma_{A}=1, \mu_{A}=0$;

2.2.3 Propose 3-Dimentional Jameel's Stressed Stock Pricing Models for IFRS 9 Compliance

Also, applying Jameel's Criterion and Jameel's Contractional-Expansional Stressed Methods to REPLACE $\left\{W_{J B}\right\}_{t \geq 0}$ of the Geometric Brownian Motion with the JAMEEL'S SUBSTITUTIONS FOR IFRS 9 COMPLIANCE for THREE-DIMENSIONAL stress closed form (1) $\left(\mu_{A} \pm \sigma_{A} W_{J B 1}(t) \pm \sigma_{A} W_{J B 2}(t) \pm\right.$ $\left.\sigma_{A} W_{J B 3}(t)\right)$, whenever $\sigma_{A}>1, \mu_{A}$ is positive infinitesimal; (2) $\left(\mu_{A} \pm W_{J B 1}(t) \pm W_{J B 2}(t) \pm W_{J B 3}(t)\right)$, whenever $\sigma_{A}=1, \mu_{A}$ is positive infinitesimal; (3) $\left( \pm \sigma_{A} W_{J B 1}(t) \pm \sigma_{A} W_{J B 2}(t) \pm \sigma_{A} W_{J B 3}(t)\right)$, whenever $\sigma_{A}>$ $1, \mu_{A}=0$; and (4) $\left( \pm W_{J B 1}(t) \pm W_{J B 2}(t) \pm W_{J B 3}(t)\right)$, whenever $\sigma_{A}=1, \mu_{A}=0$ then we have the following Propose Jameel's Stressed Closed Form Stocks Pricing Models TYPES for IFRS 9 Compliance as:

TYPE 1:

$\left(S_{J B}(t)\right)_{\text {Stressed }}=p_{0} \exp \left(\mu t+\sigma\left(\mu_{A} \pm \sigma_{A} W_{J B 1}(t) \pm \sigma_{A} W_{J B 2}(t) \pm \sigma_{A} W_{J B 3}(t)\right)\right)$,

whenever $\sigma_{A}>1, \mu_{A}$ is positive infinitesimal;

TYPE 2:

$\left(S_{J B}(t)\right)_{\text {Stressed }}=p_{0} \exp \left(\mu t+\sigma\left(\mu_{A} \pm W_{J B 1}(t) \pm W_{J B 2}(t) \pm W_{J B 3}(t)\right)\right)$,

whenever $\sigma_{A}=1, \mu_{A}$ is positive infinitesimal;

TYPE 3:

$\left(S_{J B}(t)\right)_{\text {stressed }}=p_{0} \exp \left(\mu t+\sigma\left( \pm \sigma_{A} W_{J B 1}(t) \pm \sigma_{A} W_{J B 2}(t) \pm \sigma_{A} W_{J B 3}(t)\right)\right)$,

whenever $\sigma_{A}>1, \mu_{A}=0$;

TYPE 4:

$\left(S_{J B}(t)\right)_{\text {Stressed }}=p_{0} \exp \left(\mu t+\sigma\left( \pm W_{J B 1}(t) \pm W_{J B 2}(t) \pm W_{J B 3}(t)\right)\right)$,

whenever $\sigma_{A}=1, \mu_{A}=0$; 




Figure 2. Jameel's Transformational Diagram for IFRS 9 Compliance

Generally, using Jameel's Criterion and Jameel's Contractional-Expansional Stressed Methods, we replaces the WIENER PROCESSES (NORMAL and or LOG-NORMAL) terms appears in the CLOSED FORM SOLUTIONS of Ornstein - Uhlenbeck Process, Cox-Ingersoll-Ross (1985) Model, Vasicek Model, BlackKarasinki (1991) Model, Chen (1994) Model, Kalotay - Williams - Fabozzi (1993) Model, Longstaff - Schwatz (1992) Model, Ho-Lee Model (1986) Model, Hull-White (1990) Model, Black-Derman-Toy (1990) Model, Heston Volatility Model and ETFs and Leveraged ETFs Models by JAMEEL'S SUBSTITUTIONS FOR IFRS 9 COMPLIANCE for TWO and THREE-DIMENSIONAL as presented in the case GEOMETRIC BROWNIAN MOTION of STOCKS PRICING MODELS to obtain their Stressed Closed Form Models TYPES for IFRS 9 Compliance.

\section{Results}

To test the performances of the proposed Sixteen (16) Jameel's Stressed Closed Form Solutions considering Stocks Geometric Brownian Model, the Author considered Chevron Corporation (CVX) Stock data extracted from yahoo finance using Time Series from 2014 - 1991. Thus, the data distribution Mean equal 0.000326, Standard Deviation 
equal 0.015761, the Annual drift of the year preceding 2014 (2013) equal 0.000466 and the Annual Volatility of the year preceding 2014 (2013) equal 0.008325 . Hence, $\mu_{\text {daily }}=0.000466 / 252=1.84921 E-06, \sigma_{\text {daily }}=$ $0.008325 / \sqrt{252}=0.000524$.

Therefore, $\quad \mu=\mu_{\text {daily }}-\frac{1}{2} \sigma_{\text {daily }}^{2}=1.84921 E-06-\frac{1}{2}(0.000524)^{2}=1.71192 E-06$ and $\sigma=0.000524$

while $\mu_{A}=0.030383975$, and $\sigma_{A}=0.111414539$.

The Author uses $p_{0}=108.87$ as of 11/28/2014 (a day before 12/1/2014) as the Initial Stock Price with intention to Predict Twenty One (21) working days (from 12/1/ 2014 to 12/30/ 2014) Chevron Corporation (CVX) Stock Prices thereby comparing the REAL PRICES, NORMAL PRICES with the other PROPOSED JAMEEL'S STRESSED CLOSED FORM PRICES.

The Author performs the PREDICTION Using MICROSOFT EXCEL and obtained the following RESULTS as shown in Tables and Charts below:

Note that in Table 1, the notation $\boldsymbol{T}_{\mathbf{1}}( \pm, \mp)$ means different models of Type 1, 2-Dimensional Jameel's Stressed Closed Form Prices; in Table 2, $\boldsymbol{T}_{\mathbf{2}}( \pm, \mp)$ means different models of Type 2, 2-Dimensional Jameel's Stressed Closed Form Prices; in Table 3, $\boldsymbol{T}_{\mathbf{3}}( \pm, \mp)$ means different models of Type 3, 2-Dimensional Jameel's Stressed Closed Form Prices; in Table 3, $\boldsymbol{T}_{\mathbf{4}}( \pm, \mp)$ means different models of Type 4, 2-Dimensional Jameel's Stressed Closed Form Prices; while in Table 5, the notation $\boldsymbol{T}_{\mathbf{1}}( \pm, \mp, \pm)$ means different models of Type 1, 3Dimensional Jameel's Stressed Closed Form Prices; in Table 6, $\boldsymbol{T}_{2}( \pm, \mp, \pm)$ means different models of Type 2, 3-Dimensional Jameel's Stressed Closed Form Prices; in Table 7, $\boldsymbol{T}_{3}( \pm, \bar{\mp}, \pm)$ means different models of Type 3, 3-Dimensional Jameel's Stressed Closed Form Prices; in Table 8, $\boldsymbol{T}_{\mathbf{4}}( \pm, \mp, \pm)$ means different models of Type 4, 3-Dimensional Jameel's Stressed Closed Form Prices.

Table 1. CVX Stressed Prices of Propose Type 1, 2-Dimensional Jameel's Stressed Models for IFRS 9 Compliance

\begin{tabular}{|c|c|c|c|c|c|c|c|}
\hline Date & $\mathbf{t}$ & REAL PRICES & NORMAL PRICES & $\mathbf{T 1}(+,+)$ & T1(+,-) & T1 $(-,+)$ & T1(-,-) \\
\hline $11 / 28 / 2014$ & $\mathbf{0}$ & 108.87 & & & & & \\
\hline $12 / 1 / 2014$ & 1 & 111.730003 & 108.8701864 & 108.87198 & 108.87198 & 108.87186 & 108.87186 \\
\hline $12 / 2 / 2014$ & 2 & 114.019997 & 111.7303855 & 111.73222 & 111.73222 & 111.73211 & 111.73211 \\
\hline $12 / 3 / 2014$ & 3 & 113.709999 & 114.0205826 & 114.02246 & 114.02246 & 114.02234 & 114.02234 \\
\hline $12 / 4 / 2014$ & 4 & 112.279999 & 113.7107777 & 113.71265 & 113.71265 & 113.71253 & 113.71253 \\
\hline $12 / 5 / 2014$ & 5 & 110.870003 & 112.2809601 & 112.28281 & 112.28281 & 112.28269 & 112.28269 \\
\hline $12 / 8 / 2014$ & 6 & 106.800003 & 110.8711418 & 110.87296 & 110.87296 & 110.87285 & 110.87285 \\
\hline $12 / 9 / 2014$ & 7 & 107.010002 & 106.8012828 & 106.80304 & 106.80304 & 106.80293 & 106.80293 \\
\hline $12 / 10 / 2014$ & 8 & 104.860001 & 107.0114676 & 107.01323 & 107.01323 & 107.01311 & 107.01311 \\
\hline $12 / 11 / 2014$ & 9 & 104.910004 & 104.8616166 & 104.86334 & 104.86334 & 104.86323 & 104.86323 \\
\hline $12 / 12 / 2014$ & 10 & 102.379997 & 104.9118 & 104.91353 & 104.91353 & 104.91341 & 104.91341 \\
\hline $12 / 15 / 2014$ & 11 & 100.860001 & 102.3819249 & 102.38361 & 102.38361 & 102.3835 & 102.3835 \\
\hline $12 / 16 / 2014$ & 12 & 101.699997 & 100.862073 & 100.86373 & 100.86373 & 100.86362 & 100.86362 \\
\hline $12 / 17 / 2014$ & 13 & 106.019997 & 101.7022604 & 101.70393 & 101.70393 & 101.70382 & 101.70382 \\
\hline $12 / 18 / 2014$ & 14 & 109.029999 & 106.022538 & 106.02428 & 106.02428 & 106.02417 & 106.02417 \\
\hline $12 / 19 / 2014$ & 15 & 112.93 & 109.0327988 & 109.03459 & 109.03459 & 109.03448 & 109.03448 \\
\hline $12 / 22 / 2014$ & 16 & 112.029999 & 112.9330933 & 112.93495 & 112.93495 & 112.93483 & 112.93483 \\
\hline $12 / 23 / 2014$ & 17 & 113.949997 & 112.0332594 & 112.0351 & 112.0351 & 112.03499 & 112.03499 \\
\hline $12 / 24 / 2014$ & 18 & 113.470001 & 113.9535084 & 113.95538 & 113.95538 & 113.95526 & 113.95526 \\
\hline $12 / 26 / 2014$ & 19 & 113.25 & 113.4736918 & 113.47556 & 113.47556 & 113.47544 & 113.47544 \\
\hline $12 / 29 / 2014$ & 20 & 113.32 & 113.2538776 & 113.25574 & 113.25574 & 113.25562 & 113.25562 \\
\hline $12 / 30 / 2014$ & 21 & 113.110001 & 113.324074 & 113.32594 & 113.32594 & 113.32582 & 113.32582 \\
\hline
\end{tabular}


Table 2. CVX Stressed Prices of Propose Type 2, 2-Dimensional Jameel's Stressed Models for IFRS 9 Compliance

\begin{tabular}{llllllll}
\hline Date & t & REAL PRICES & NORMAL PRICES & T2(+,+) & T2(+,-) & T2(-,+) & T2(-,-) \\
\hline $11 / 28 / 2014$ & $\mathbf{0}$ & $\mathbf{1 0 8 . 8 7}$ & & & & & \\
$12 / 1 / 2014$ & 1 & 111.730003 & 108.8701864 & 108.87243 & 108.87243 & 108.87141 & 108.87141 \\
$12 / 2 / 2014$ & 2 & 114.019997 & 111.7303855 & 111.73268 & 111.73268 & 111.73165 & 111.73165 \\
$12 / 3 / 2014$ & 3 & 113.709999 & 114.0205826 & 114.02292 & 114.02292 & 114.02187 & 114.02187 \\
$12 / 4 / 2014$ & 4 & 112.279999 & 113.7107777 & 113.71311 & 113.71311 & 113.71206 & 113.71206 \\
$12 / 5 / 2014$ & 5 & 110.870003 & 112.2809601 & 112.28327 & 112.28327 & 112.28223 & 112.28223 \\
$12 / 8 / 2014$ & 6 & 106.800003 & 110.8711418 & 110.87342 & 110.87342 & 110.87239 & 110.87239 \\
$12 / 9 / 2014$ & 7 & 107.010002 & 106.8012828 & 106.80349 & 106.80349 & 106.80248 & 106.80248 \\
$12 / 10 / 2014$ & 8 & 104.860001 & 107.0114676 & 107.01368 & 107.01368 & 107.01266 & 107.01266 \\
$12 / 11 / 2014$ & 9 & 104.910004 & 104.8616166 & 104.86379 & 104.86379 & 104.86278 & 104.86278 \\
$12 / 12 / 2014$ & 10 & 102.379997 & 104.9118 & 104.91397 & 104.91397 & 104.91297 & 104.91297 \\
$12 / 15 / 2014$ & 11 & 100.860001 & 102.3819249 & 102.38405 & 102.38405 & 102.38306 & 102.38306 \\
$12 / 16 / 2014$ & 12 & 101.699997 & 100.862073 & 100.86417 & 100.86417 & 100.86319 & 100.86319 \\
$12 / 17 / 2014$ & 13 & 106.019997 & 101.7022604 & 101.70437 & 101.70437 & 101.70339 & 101.70339 \\
$12 / 18 / 2014$ & 14 & 109.029999 & 106.022538 & 106.02473 & 106.02473 & 106.02372 & 106.02372 \\
$12 / 19 / 2014$ & 15 & 112.93 & 109.0327988 & 109.03505 & 109.03505 & 109.03402 & 109.03402 \\
$12 / 22 / 2014$ & 16 & 112.029999 & 112.9330933 & 112.93541 & 112.93541 & 112.93437 & 112.93437 \\
$12 / 23 / 2014$ & 17 & 113.949997 & 112.0332594 & 112.03556 & 112.03556 & 112.03452 & 112.03452 \\
$12 / 24 / 2014$ & 18 & 113.470001 & 113.9535084 & 113.95585 & 113.95585 & 113.9548 & 113.9548 \\
$12 / 26 / 2014$ & 19 & 113.25 & 113.4736918 & 113.47602 & 113.47602 & 113.47497 & 113.47497 \\
$12 / 29 / 2014$ & 20 & 113.32 & 113.2538776 & 113.2562 & 113.2562 & 113.25516 & 113.25516 \\
$12 / 30 / 2014$ & 21 & 113.110001 & 113.324074 & 113.3264 & 113.3264 & 113.32535 & 113.32535 \\
\hline
\end{tabular}

Table 3. CVX Stressed Prices of Propose Type 3, 2-Dimensional Jameel's Stressed Models for IFRS 9 Compliance

\begin{tabular}{llllllll}
\hline Date & $\mathbf{t}$ & REAL PRICES & \multicolumn{1}{l}{ NORMAL PRICES } & T3(+,+) & T3(+,-) & T3(-,+) & T3(-,-) \\
\hline $11 / 28 / 2014$ & $\mathbf{0}$ & $\mathbf{1 0 8 . 8 7}$ & & & & & \\
$12 / 1 / 2014$ & 1 & 111.730003 & 108.8701864 & 108.87024 & 108.87024 & 108.87013 & 108.87013 \\
$12 / 2 / 2014$ & 2 & 114.019997 & 111.7303855 & 111.73044 & 111.73044 & 111.73033 & 111.73033 \\
$12 / 3 / 2014$ & 3 & 113.709999 & 114.0205826 & 114.02064 & 114.02064 & 114.02052 & 114.02052 \\
$12 / 4 / 2014$ & 4 & 112.279999 & 113.7107777 & 113.71084 & 113.71084 & 113.71072 & 113.71072 \\
$12 / 5 / 2014$ & 5 & 110.870003 & 112.2809601 & 112.28102 & 112.28102 & 112.2809 & 112.2809 \\
$12 / 8 / 2014$ & 6 & 106.800003 & 110.8711418 & 110.8712 & 110.8712 & 110.87108 & 110.87108 \\
$12 / 9 / 2014$ & 7 & 107.010002 & 106.8012828 & 106.80134 & 106.80134 & 106.80123 & 106.80123 \\
$12 / 10 / 2014$ & 8 & 104.860001 & 107.0114676 & 107.01152 & 107.01152 & 107.01141 & 107.01141 \\
$12 / 11 / 2014$ & 9 & 104.910004 & 104.8616166 & 104.86167 & 104.86167 & 104.86156 & 104.86156 \\
$12 / 12 / 2014$ & 10 & 102.379997 & 104.9118 & 104.91186 & 104.91186 & 104.91174 & 104.91174 \\
$12 / 15 / 2014$ & 11 & 100.860001 & 102.3819249 & 102.38198 & 102.38198 & 102.38187 & 102.38187 \\
$12 / 16 / 2014$ & 12 & 101.699997 & 100.862073 & 100.86213 & 100.86213 & 100.86202 & 100.86202 \\
$12 / 17 / 2014$ & 13 & 106.019997 & 101.7022604 & 101.70232 & 101.70232 & 101.70221 & 101.70221 \\
$12 / 18 / 2014$ & 14 & 109.029999 & 106.022538 & 106.02259 & 106.02259 & 106.02248 & 106.02248 \\
$12 / 19 / 2014$ & 15 & 112.93 & 109.0327988 & 109.03286 & 109.03286 & 109.03274 & 109.03274 \\
$12 / 22 / 2014$ & 16 & 112.029999 & 112.9330933 & 112.93315 & 112.93315 & 112.93304 & 112.93304 \\
$12 / 23 / 2014$ & 17 & 113.949997 & 112.0332594 & 112.03332 & 112.03332 & 112.0332 & 112.0332 \\
$12 / 24 / 2014$ & 18 & 113.470001 & 113.9535084 & 113.95357 & 113.95357 & 113.95345 & 113.95345 \\
$12 / 26 / 2014$ & 19 & 113.25 & 113.4736918 & 113.47375 & 113.47375 & 113.47363 & 113.47363 \\
$12 / 29 / 2014$ & 20 & 113.32 & 113.2538776 & 113.25394 & 113.25394 & 113.25382 & 113.25382 \\
$12 / 30 / 2014$ & 21 & 113.110001 & 113.324074 & 113.32413 & 113.32413 & 113.32402 & 113.32402 \\
\hline
\end{tabular}


Table 4. CVX Stressed Prices of Propose Type 4, 2-Dimensional Jameel's Stressed Models for IFRS 9 Compliance

\begin{tabular}{|c|c|c|c|c|c|c|c|}
\hline Date & $\mathbf{t}$ & REAL PRICES & NORMAL PRICES & $\mathrm{T} 4(+,+)$ & T4(+,-) & T4 $(-,+)$ & T4(-,-) \\
\hline $11 / 28 / 2014$ & $\mathbf{0}$ & 108.87 & & & & & \\
\hline $12 / 1 / 2014$ & 1 & 111.730003 & 108.8701864 & 108.8707 & 108.8707 & 108.86967 & 108.86967 \\
\hline $12 / 2 / 2014$ & 2 & 114.019997 & 111.7303855 & 111.7309 & 111.7309 & 111.72987 & 111.72987 \\
\hline $12 / 3 / 2014$ & 3 & 113.709999 & 114.0205826 & 114.02111 & 114.02111 & 114.02006 & 114.02006 \\
\hline $12 / 4 / 2014$ & 4 & 112.279999 & 113.7107777 & 113.7113 & 113.7113 & 113.71025 & 113.71025 \\
\hline $12 / 5 / 2014$ & 5 & 110.870003 & 112.2809601 & 112.28148 & 112.28148 & 112.28044 & 112.28044 \\
\hline $12 / 8 / 2014$ & 6 & 106.800003 & 110.8711418 & 110.87166 & 110.87166 & 110.87062 & 110.87062 \\
\hline $12 / 9 / 2014$ & 7 & 107.010002 & 106.8012828 & 106.80179 & 106.80179 & 106.80078 & 106.80078 \\
\hline $12 / 10 / 2014$ & 8 & 104.860001 & 107.0114676 & 107.01197 & 107.01197 & 107.01096 & 107.01096 \\
\hline $12 / 11 / 2014$ & 9 & 104.910004 & 104.8616166 & 104.86212 & 104.86212 & 104.86112 & 104.86112 \\
\hline $12 / 12 / 2014$ & 10 & 102.379997 & 104.9118 & 104.9123 & 104.9123 & 104.9113 & 104.9113 \\
\hline $12 / 15 / 2014$ & 11 & 100.860001 & 102.3819249 & 102.38242 & 102.38242 & 102.38143 & 102.38143 \\
\hline $12 / 16 / 2014$ & 12 & 101.699997 & 100.862073 & 100.86256 & 100.86256 & 100.86158 & 100.86158 \\
\hline $12 / 17 / 2014$ & 13 & 106.019997 & 101.7022604 & 101.70275 & 101.70275 & 101.70177 & 101.70177 \\
\hline $12 / 18 / 2014$ & 14 & 109.029999 & 106.022538 & 106.02304 & 106.02304 & 106.02203 & 106.02203 \\
\hline $12 / 19 / 2014$ & 15 & 112.93 & 109.0327988 & 109.03331 & 109.03331 & 109.03229 & 109.03229 \\
\hline $12 / 22 / 2014$ & 16 & 112.029999 & 112.9330933 & 112.93362 & 112.93362 & 112.93257 & 112.93257 \\
\hline $12 / 23 / 2014$ & 17 & 113.949997 & 112.0332594 & 112.03378 & 112.03378 & 112.03274 & 112.03274 \\
\hline $12 / 24 / 2014$ & 18 & 113.470001 & 113.9535084 & 113.95403 & 113.95403 & 113.95298 & 113.95298 \\
\hline $12 / 26 / 2014$ & 19 & 113.25 & 113.4736918 & 113.47422 & 113.47422 & 113.47317 & 113.47317 \\
\hline $12 / 29 / 2014$ & 20 & 113.32 & 113.2538776 & 113.2544 & 113.2544 & 113.25335 & 113.25335 \\
\hline $12 / 30 / 2014$ & 21 & 113.110001 & 113.324074 & 113.3246 & 113.3246 & 113.32355 & 113.32355 \\
\hline
\end{tabular}

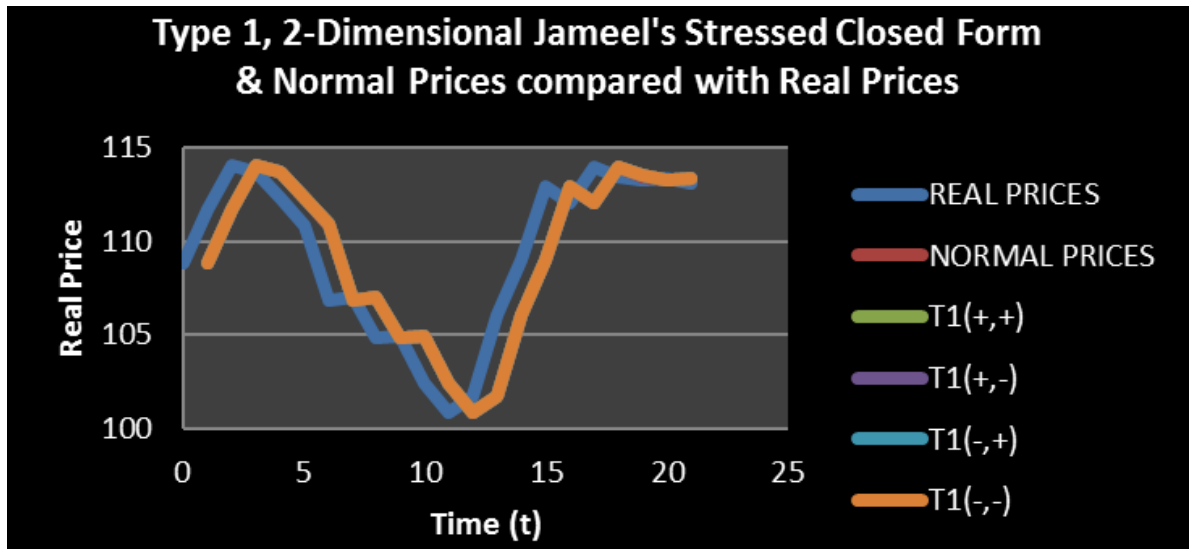

Figure 3.

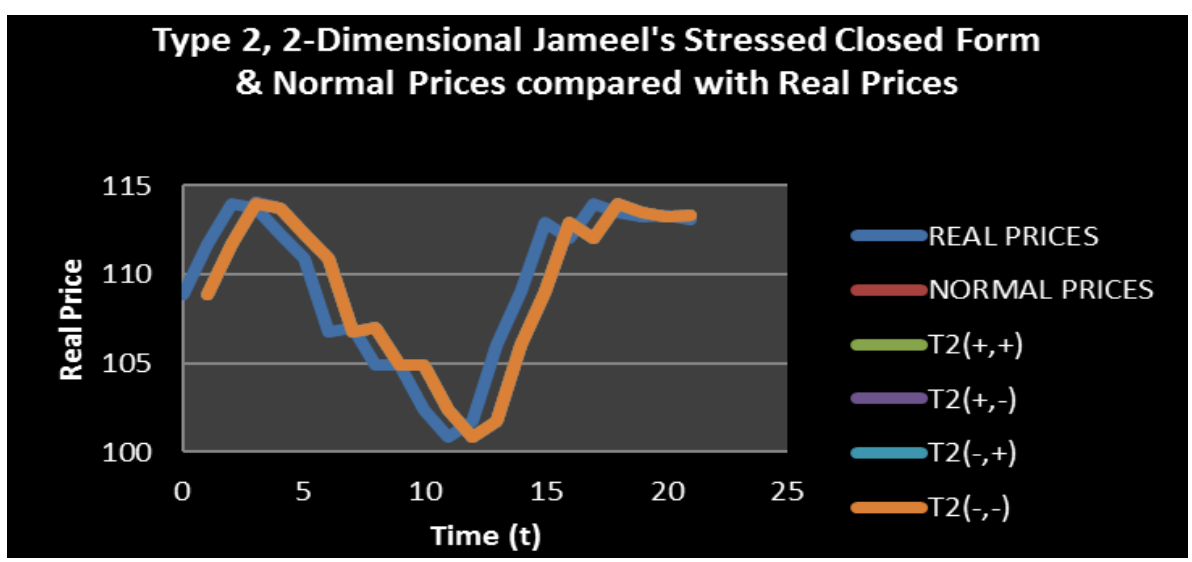

Figure 4. 


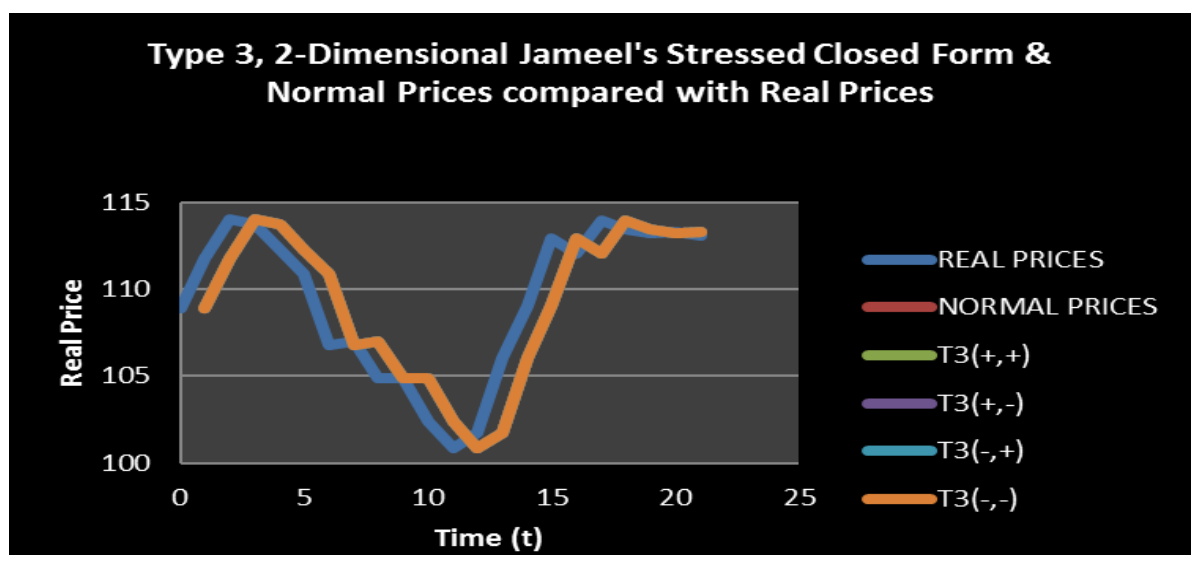

Figure 5.

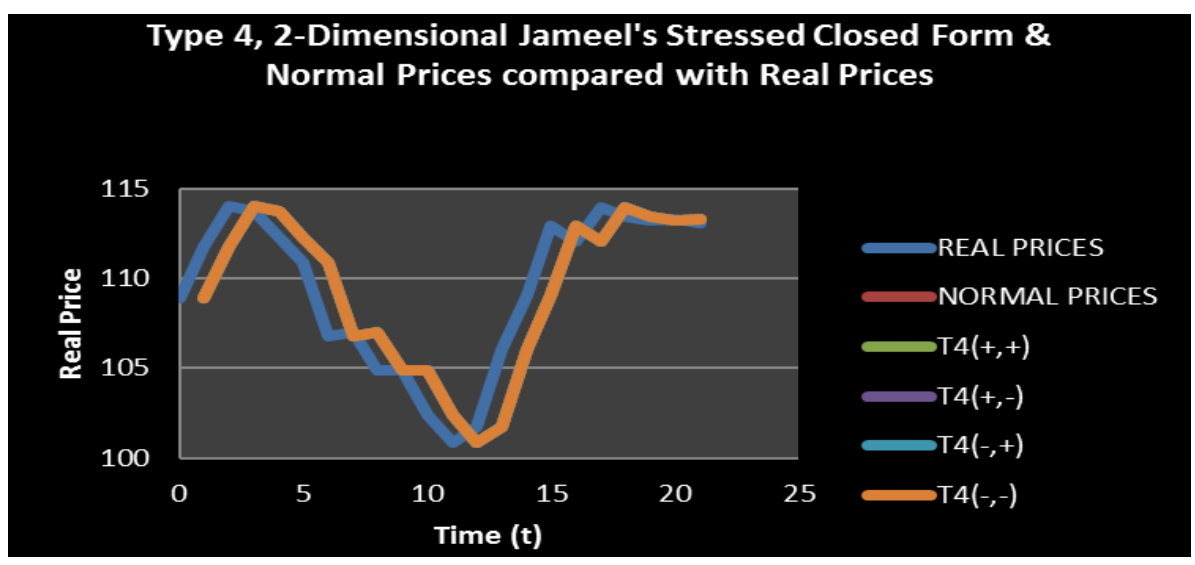

Figure 6.

Table 5. CVX Stressed Prices of Propose Type 1, 3-Dimensional Jameel's Stressed Models for IFRS 9 Compliance

\begin{tabular}{|c|c|c|c|c|c|c|c|c|c|c|c|}
\hline Date & $\mathbf{t}$ & $\begin{array}{l}\text { REAL } \\
\text { PRICES }\end{array}$ & $\begin{array}{l}\text { NORMAL } \\
\text { PRICES }\end{array}$ & $\mathbf{T} 1(+,+,+)$ & $\mathrm{T} 1(+,+,-)$ & T1 $(+,-,-)$ & T1(-,-,-) & T1 $(-,-,+)$ & $\mathbf{T} 1(-,+,+)$ & T1(-,+,-) & $\mathbf{T} 1(+,-,+)$ \\
\hline $11 / 28 / 2014$ & $\mathbf{0}$ & 108.87 & & & & & & & & & \\
\hline $12 / 1 / 2014$ & 1 & 111.730003 & 108.8701864 & 108.87198 & 108.87198 & 108.87198 & 108.87186 & 108.87186 & 108.87186 & 108.87186 & 108.87198 \\
\hline $12 / 2 / 2014$ & 2 & 114.019997 & 111.7303855 & 111.73222 & 111.73222 & 111.73222 & 111.73211 & 111.73211 & 111.73211 & 111.73211 & 111.73222 \\
\hline $12 / 3 / 2014$ & 3 & 113.709999 & 114.0205826 & 114.02246 & 114.02246 & 114.02246 & 114.02234 & 114.02234 & 114.02234 & 114.02234 & 114.02246 \\
\hline $12 / 4 / 2014$ & 4 & 112.279999 & 113.7107777 & 113.71265 & 113.71265 & 113.71265 & 113.71253 & 113.71253 & 113.71253 & 113.71253 & 113.71265 \\
\hline $12 / 5 / 2014$ & 5 & 110.870003 & 112.2809601 & 112.28281 & 112.28281 & 112.28281 & 112.28269 & 112.28269 & 112.28269 & 112.28269 & 112.28281 \\
\hline $12 / 8 / 2014$ & 6 & 106.800003 & 110.8711418 & 110.87296 & 110.87296 & 110.87296 & 110.87285 & 110.87285 & 110.87285 & 110.87285 & 110.87296 \\
\hline $12 / 9 / 2014$ & 7 & 107.010002 & 106.8012828 & 106.80304 & 106.80304 & 106.80304 & 106.80293 & 106.80293 & 106.80293 & 106.80293 & 106.80304 \\
\hline $12 / 10 / 2014$ & 8 & 104.860001 & 107.0114676 & 107.01323 & 107.01323 & 107.01323 & 107.01311 & 107.01311 & 107.01311 & 107.01311 & 107.01323 \\
\hline $12 / 11 / 2014$ & 9 & 104.910004 & 104.8616166 & 104.86334 & 104.86334 & 104.86334 & 104.86323 & 104.86323 & 104.86323 & 104.86323 & 104.86334 \\
\hline $12 / 12 / 2014$ & 10 & 102.379997 & 104.9118 & 104.91353 & 104.91353 & 104.91353 & 104.91341 & 104.91341 & 104.91341 & 104.91341 & 104.91353 \\
\hline $12 / 15 / 2014$ & 11 & 100.860001 & 102.3819249 & 102.38361 & 102.38361 & 102.38361 & 102.3835 & 102.3835 & 102.3835 & 102.3835 & 102.38361 \\
\hline $12 / 16 / 2014$ & 12 & 101.699997 & 100.862073 & 100.86373 & 100.86373 & 100.86373 & 100.86362 & 100.86362 & 100.86362 & 100.86362 & 100.86373 \\
\hline $12 / 17 / 2014$ & 13 & 106.019997 & 101.7022604 & 101.70393 & 101.70393 & 101.70393 & 101.70382 & 101.70382 & 101.70382 & 101.70382 & 101.70393 \\
\hline $12 / 18 / 2014$ & 14 & 109.029999 & 106.022538 & 106.02428 & 106.02428 & 106.02428 & 106.02417 & 106.02417 & 106.02417 & 106.02417 & 106.02428 \\
\hline $12 / 19 / 2014$ & 15 & 112.93 & 109.0327988 & 109.03459 & 109.03459 & 109.03459 & 109.03448 & 109.03448 & 109.03448 & 109.03448 & 109.03459 \\
\hline $12 / 22 / 2014$ & 16 & 112.029999 & 112.9330933 & 112.93495 & 112.93495 & 112.93495 & 112.93483 & 112.93483 & 112.93483 & 112.93483 & 112.93495 \\
\hline $12 / 23 / 2014$ & 17 & 113.949997 & 112.0332594 & 112.0351 & 112.0351 & 112.0351 & 112.03499 & 112.03499 & 112.03499 & 112.03499 & 112.0351 \\
\hline $12 / 24 / 2014$ & 18 & 113.470001 & 113.9535084 & 113.95538 & 113.95538 & 113.95538 & 113.95526 & 113.95526 & 113.95526 & 113.95526 & 113.95538 \\
\hline $12 / 26 / 2014$ & 19 & 113.25 & 113.4736918 & 113.47556 & 113.47556 & 113.47556 & 113.47544 & 113.47544 & 113.47544 & 113.47544 & 113.47556 \\
\hline $12 / 29 / 2014$ & 20 & 113.32 & 113.2538776 & 113.25574 & 113.25574 & 113.25574 & 113.25562 & 113.25562 & 113.25562 & 113.25562 & 113.25574 \\
\hline $12 / 30 / 2014$ & 21 & 113.110001 & 113.324074 & 113.32594 & 113.32594 & 113.32594 & 113.32582 & 113.32582 & 113.32582 & 113.32582 & 113.32594 \\
\hline
\end{tabular}


Table 6. CVX Stressed Prices of Propose Type 2, 3-Dimensional Jameel's Stressed Models for IFRS 9 Compliance

\begin{tabular}{|c|c|c|c|c|c|c|c|c|c|c|c|}
\hline Date & $\mathbf{t}$ & $\begin{array}{l}\text { REAL } \\
\text { PRICES }\end{array}$ & $\begin{array}{l}\text { NORMAL } \\
\text { PRICES } \\
\end{array}$ & $\mathbf{T} 2(+,+,+)$ & $\mathrm{T} 2(+,+,-)$ & $\mathrm{T2}(+,-,-)$ & T2(-,-,-) & T2(-,-,+) & T2(-,+,+) & T2(-,+,-) & T2(+,-,+) \\
\hline $11 / 28 / 2014$ & $\mathbf{0}$ & 108.87 & & & & & & & & & \\
\hline $12 / 1 / 2014$ & 1 & 111.730003 & 108.8701864 & 108.87243 & 108.87243 & 108.87243 & 108.87141 & 108.87141 & 108.87141 & 108.87141 & 108.87243 \\
\hline $12 / 2 / 2014$ & 2 & 114.019997 & 111.7303855 & 111.73268 & 111.73268 & 111.73268 & 111.73164 & 111.73165 & 111.73165 & 111.73164 & 111.73268 \\
\hline $12 / 3 / 2014$ & 3 & 113.709999 & 114.0205826 & 114.02292 & 114.02292 & 114.02292 & 114.02187 & 114.02187 & 114.02187 & 114.02187 & 114.02292 \\
\hline $12 / 4 / 2014$ & 4 & 112.279999 & 113.7107777 & 113.71311 & 113.71311 & 113.71311 & 113.71206 & 113.71206 & 113.71206 & 113.71206 & 113.71311 \\
\hline $12 / 5 / 2014$ & 5 & 110.870003 & 112.2809601 & 112.28327 & 112.28327 & 112.28327 & 112.28223 & 112.28223 & 112.28223 & 112.28223 & 112.28327 \\
\hline $12 / 8 / 2014$ & 6 & 106.800003 & 110.8711418 & 110.87342 & 110.87342 & 110.87342 & 110.87239 & 110.87239 & 110.87239 & 110.87239 & 110.87342 \\
\hline $12 / 9 / 2014$ & 7 & 107.010002 & 106.8012828 & 106.80349 & 106.80349 & 106.80349 & 106.80248 & 106.80248 & 106.80248 & 106.80248 & 106.80349 \\
\hline $12 / 10 / 2014$ & 8 & 104.860001 & 107.0114676 & 107.01368 & 107.01368 & 107.01368 & 107.01266 & 107.01266 & 107.01266 & 107.01266 & 107.01368 \\
\hline $12 / 11 / 2014$ & 9 & 104.910004 & 104.8616166 & 104.86379 & 104.86379 & 104.86379 & 104.86278 & 104.86278 & 104.86278 & 104.86278 & 104.86379 \\
\hline $12 / 12 / 2014$ & 10 & 102.379997 & 104.9118 & 104.91397 & 104.91397 & 104.91397 & 104.91297 & 104.91297 & 104.91297 & 104.91297 & 104.91397 \\
\hline $12 / 15 / 2014$ & 11 & 100.860001 & 102.3819249 & 102.38405 & 102.38405 & 102.38405 & 102.38306 & 102.38306 & 102.38306 & 102.38306 & 102.38405 \\
\hline $12 / 16 / 2014$ & 12 & 101.699997 & 100.862073 & 100.86417 & 100.86417 & 100.86417 & 100.86319 & 100.86319 & 100.86319 & 100.86319 & 100.86417 \\
\hline $12 / 17 / 2014$ & 13 & 106.019997 & 101.7022604 & 101.70437 & 101.70437 & 101.70437 & 101.70339 & 101.70339 & 101.70339 & 101.70339 & 101.70437 \\
\hline $12 / 18 / 2014$ & 14 & 109.029999 & 106.022538 & 106.02473 & 106.02473 & 106.02473 & 106.02372 & 106.02372 & 106.02372 & 106.02372 & 106.02473 \\
\hline $12 / 19 / 2014$ & 15 & 112.93 & 109.0327988 & 109.03505 & 109.03505 & 109.03505 & 109.03402 & 109.03402 & 109.03402 & 109.03402 & 109.03505 \\
\hline $12 / 22 / 2014$ & 16 & 112.029999 & 112.9330933 & 112.93541 & 112.93541 & 112.93541 & 112.93437 & 112.93437 & 112.93437 & 112.93437 & 112.93541 \\
\hline $12 / 23 / 2014$ & 17 & 113.949997 & 112.0332594 & 112.03556 & 112.03556 & 112.03556 & 112.03452 & 112.03452 & 112.03452 & 112.03452 & 112.03556 \\
\hline $12 / 24 / 2014$ & 18 & 113.470001 & 113.9535084 & 113.95585 & 113.95585 & 113.95585 & 113.9548 & 113.9548 & 113.9548 & 113.9548 & 113.95585 \\
\hline $12 / 26 / 2014$ & 19 & 113.25 & 113.4736918 & 113.47602 & 113.47602 & 113.47602 & 113.47497 & 113.47497 & 113.47497 & 113.47497 & 113.47602 \\
\hline $12 / 29 / 2014$ & 20 & 113.32 & 113.2538776 & 113.2562 & 113.2562 & 113.2562 & 113.25516 & 113.25516 & 113.25516 & 113.25516 & 113.2562 \\
\hline $12 / 30 / 2014$ & 21 & 113.110001 & 113.324074 & 113.3264 & 113.3264 & 113.3264 & 113.32535 & 113.32535 & 113.32535 & 113.32535 & 113.3264 \\
\hline
\end{tabular}

Table 7. CVX Stressed Prices of Propose Type 3, 3-Dimensional Jameel's Stressed Models for IFRS 9 Compliance

\begin{tabular}{|c|c|c|c|c|c|c|c|c|c|c|c|}
\hline Date & $\mathbf{t}$ & $\begin{array}{l}\text { REAL } \\
\text { PRICES }\end{array}$ & $\begin{array}{l}\text { NORMAL } \\
\text { PRICES }\end{array}$ & T3(+,+,+) & T3(+,+,,-) & T3 $(+,-,-)$ & T3 (-,-,-) & T3 $(-,-,+)$ & T3 $(-,+,+)$ & T3 $(-,+,-)$ & T3 $(+,-,+)$ \\
\hline $11 / 28 / 2014$ & 0 & 108.87 & & & & & & & & & \\
\hline $12 / 1 / 2014$ & 1 & 111.730003 & 108.8701864 & 108.87024 & 108.87024 & 108.87024 & 108.87013 & 108.87013 & 108.87013 & 108.87013 & 108.87024 \\
\hline $12 / 2 / 2014$ & 2 & 114.019997 & 111.7303855 & 111.73044 & 111.73044 & 111.73044 & 111.73033 & 111.73033 & 111.73033 & 111.73033 & 111.73044 \\
\hline $12 / 3 / 2014$ & 3 & 113.709999 & 114.0205826 & 114.02064 & 114.02064 & 114.02064 & 114.02052 & 114.02052 & 114.02052 & 114.02052 & 114.02064 \\
\hline $12 / 4 / 2014$ & 4 & 112.279999 & 113.7107777 & 113.71084 & 113.71084 & 113.71084 & 113.71072 & 113.71072 & 113.71072 & 113.71072 & 113.71084 \\
\hline $12 / 5 / 2014$ & 5 & 110.870003 & 112.2809601 & 112.28102 & 112.28102 & 112.28102 & 112.2809 & 112.2809 & 112.2809 & 112.2809 & 112.28102 \\
\hline $12 / 8 / 2014$ & 6 & 106.800003 & 110.8711418 & 110.8712 & 110.8712 & 110.8712 & 110.87108 & 110.87108 & 110.87108 & 110.87108 & 110.8712 \\
\hline $12 / 9 / 2014$ & 7 & 107.010002 & 106.8012828 & 106.80134 & 106.80134 & 106.80134 & 106.80123 & 106.80123 & 106.80123 & 106.80123 & 106.80134 \\
\hline $12 / 10 / 2014$ & 8 & 104.860001 & 107.0114676 & 107.01152 & 107.01152 & 107.01152 & 107.01141 & 107.01141 & 107.01141 & 107.01141 & 107.01152 \\
\hline $12 / 11 / 2014$ & 9 & 104.910004 & 104.8616166 & 104.86167 & 104.86167 & 104.86167 & 104.86156 & 104.86156 & 104.86156 & 104.86156 & 104.86167 \\
\hline $12 / 12 / 2014$ & 10 & 102.379997 & 104.9118 & 104.91186 & 104.91186 & 104.91186 & 104.91174 & 104.91174 & 104.91174 & 104.91174 & 104.91186 \\
\hline $12 / 15 / 2014$ & 11 & 100.860001 & 102.3819249 & 102.38198 & 102.38198 & 102.38198 & 102.38187 & 102.38187 & 102.38187 & 102.38187 & 102.38198 \\
\hline $12 / 16 / 2014$ & 12 & 101.699997 & 100.862073 & 100.86213 & 100.86213 & 100.86213 & 100.86202 & 100.86202 & 100.86202 & 100.86202 & 100.86213 \\
\hline $12 / 17 / 2014$ & 13 & 106.019997 & 101.7022604 & 101.70232 & 101.70232 & 101.70232 & 101.70221 & 101.70221 & 101.70221 & 101.70221 & 101.70232 \\
\hline $12 / 18 / 2014$ & 14 & 109.029999 & 106.022538 & 106.02259 & 106.02259 & 106.02259 & 106.02248 & 106.02248 & 106.02248 & 106.02248 & 106.02259 \\
\hline $12 / 19 / 2014$ & 15 & 112.93 & 109.0327988 & 109.03286 & 109.03286 & 109.03286 & 109.03274 & 109.03274 & 109.03274 & 109.03274 & 109.03286 \\
\hline $12 / 22 / 2014$ & 16 & 112.029999 & 112.9330933 & 112.93315 & 112.93315 & 112.93315 & 112.93304 & 112.93304 & 112.93304 & 112.93304 & 112.93315 \\
\hline $12 / 23 / 2014$ & 17 & 113.949997 & 112.0332594 & 112.03332 & 112.03332 & 112.03332 & 112.0332 & 112.0332 & 112.0332 & 112.0332 & 112.03332 \\
\hline $12 / 24 / 2014$ & 18 & 113.470001 & 113.9535084 & 113.95357 & 113.95357 & 113.95357 & 113.95345 & 113.95345 & 113.95345 & 113.95345 & 113.95357 \\
\hline $12 / 26 / 2014$ & 19 & 113.25 & 113.4736918 & 113.47375 & 113.47375 & 113.47375 & 113.47363 & 113.47363 & 113.47363 & 113.47363 & 113.47375 \\
\hline $12 / 29 / 2014$ & 20 & 113.32 & 113.2538776 & 113.25394 & 113.25394 & 113.25394 & 113.25382 & 113.25382 & 113.25382 & 113.25382 & 113.25394 \\
\hline $12 / 30 / 2014$ & 21 & 113.110001 & 113.324074 & 113.32413 & 113.32413 & 113.32413 & 113.32402 & 113.32402 & 113.32402 & 113.32402 & 113.32413 \\
\hline
\end{tabular}


Table 8. CVX Stressed Prices of Propose Type 4, 3-Dimensional Jameel's Stressed Models for IFRS 9 Compliance

\begin{tabular}{|c|c|c|c|c|c|c|c|c|c|c|c|}
\hline Date & $\mathbf{t}$ & $\begin{array}{l}\text { REAL } \\
\text { PRICES }\end{array}$ & $\begin{array}{l}\text { NORMAL } \\
\text { PRICES } \\
\end{array}$ & T4 $(+,+,+)$ & T4 (+,+,-) & T4 (+,-,-) & T4 (-,-,-) & T4 $(-,-,+)$, & T4 $(-,+,+)$ & T4 (-,+,-) & T4 $(+,-,+)$ \\
\hline $11 / 28 / 2014$ & 0 & 108.87 & & & & & & & & & \\
\hline $12 / 1 / 2014$ & 1 & 111.730003 & 108.8701864 & 108.8707 & 108.8707 & 108.8707 & 108.86967 & 108.86967 & 108.86967 & 108.86967 & 108.8707 \\
\hline $12 / 2 / 2014$ & 2 & 114.019997 & 111.7303855 & 111.73091 & 111.7309 & 111.7309 & 111.72987 & 111.72987 & 111.72987 & 111.72987 & 111.73091 \\
\hline $12 / 3 / 2014$ & 3 & 113.709999 & 114.0205826 & 114.02111 & 114.02111 & 114.02111 & 114.02006 & 114.02006 & 114.02006 & 114.02006 & 114.02111 \\
\hline $12 / 4 / 2014$ & 4 & 112.279999 & 113.7107777 & 113.7113 & 113.7113 & 113.7113 & 113.71025 & 113.71025 & 113.71025 & 113.71025 & 113.7113 \\
\hline $12 / 5 / 2014$ & 5 & 110.870003 & 112.2809601 & 112.28148 & 112.28148 & 112.28148 & 112.28044 & 112.28044 & 112.28044 & 112.28044 & 112.28148 \\
\hline $12 / 8 / 2014$ & 6 & 106.800003 & 110.8711418 & 110.87166 & 110.87166 & 110.87166 & 110.87062 & 110.87062 & 110.87062 & 110.87062 & 110.87166 \\
\hline $12 / 9 / 2014$ & 7 & 107.010002 & 106.8012828 & 106.80179 & 106.80179 & 106.80179 & 106.80078 & 106.80078 & 106.80078 & 106.80078 & 106.80179 \\
\hline $12 / 10 / 2014$ & 8 & 104.860001 & 107.0114676 & 107.01197 & 107.01197 & 107.01197 & 107.01096 & 107.01096 & 107.01096 & 107.01096 & 107.01197 \\
\hline $12 / 11 / 2014$ & 9 & 104.910004 & 104.8616166 & 104.86212 & 104.86212 & 104.86212 & 104.86112 & 104.86112 & 104.86112 & 104.86112 & 104.86212 \\
\hline $12 / 12 / 2014$ & 10 & 102.379997 & 104.9118 & 104.9123 & 104.9123 & 104.9123 & 104.9113 & 104.9113 & 104.9113 & 104.9113 & 104.9123 \\
\hline $12 / 15 / 2014$ & 11 & 100.860001 & 102.3819249 & 102.38242 & 102.38242 & 102.38242 & 102.38143 & 102.38143 & 102.38143 & 102.38143 & 102.38242 \\
\hline $12 / 16 / 2014$ & 12 & 101.699997 & 100.862073 & 100.86256 & 100.86256 & 100.86256 & 100.86158 & 100.86158 & 100.86158 & 100.86158 & 100.86256 \\
\hline $12 / 17 / 2014$ & 13 & 106.019997 & 101.7022604 & 101.70275 & 101.70275 & 101.70275 & 101.70177 & 101.70177 & 101.70177 & 101.70177 & 101.70275 \\
\hline $12 / 18 / 2014$ & 14 & 109.029999 & 106.022538 & 106.02304 & 106.02304 & 106.02304 & 106.02203 & 106.02203 & 106.02203 & 106.02203 & 106.02304 \\
\hline $12 / 19 / 2014$ & 15 & 112.93 & 109.0327988 & 109.03331 & 109.03331 & 109.03331 & 109.03229 & 109.03229 & 109.03229 & 109.03229 & 109.03331 \\
\hline $12 / 22 / 2014$ & 16 & 112.029999 & 112.9330933 & 112.93362 & 112.93362 & 112.93362 & 112.93257 & 112.93257 & 112.93257 & 112.93257 & 112.93362 \\
\hline $12 / 23 / 2014$ & 17 & 113.949997 & 112.0332594 & 112.03378 & 112.03378 & 112.03378 & 112.03274 & 112.03274 & 112.03274 & 112.03274 & 112.03378 \\
\hline $12 / 24 / 2014$ & 18 & 113.470001 & 113.9535084 & 113.95403 & 113.95403 & 113.95403 & 113.95298 & 113.95298 & 113.95298 & 113.95298 & 113.95403 \\
\hline $12 / 26 / 2014$ & 19 & 113.25 & 113.4736918 & 113.47422 & 113.47422 & 113.47422 & 113.47317 & 113.47317 & 113.47317 & 113.47317 & 113.47422 \\
\hline $12 / 29 / 2014$ & 20 & 113.32 & 113.2538776 & 113.2544 & 113.2544 & 113.2544 & 113.25335 & 113.25335 & 113.25335 & 113.25335 & 113.2544 \\
\hline $12 / 30 / 2014$ & 21 & 113.110001 & 113.324074 & 113.3246 & 113.3246 & 113.3246 & 113.32355 & 113.32355 & 113.32355 & 113.32355 & 113.3246 \\
\hline
\end{tabular}

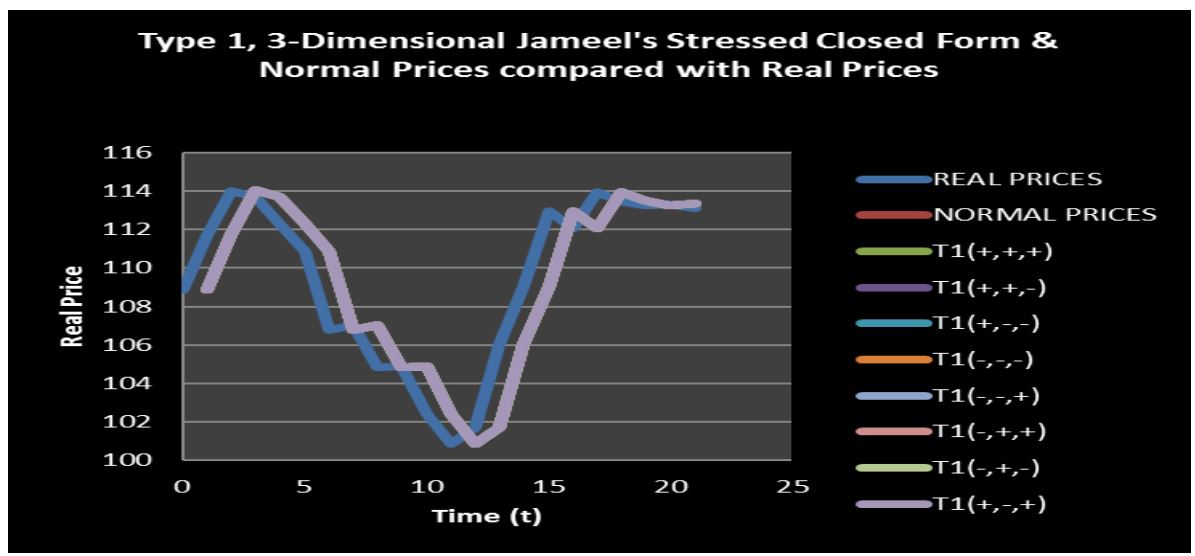

Figure 7.

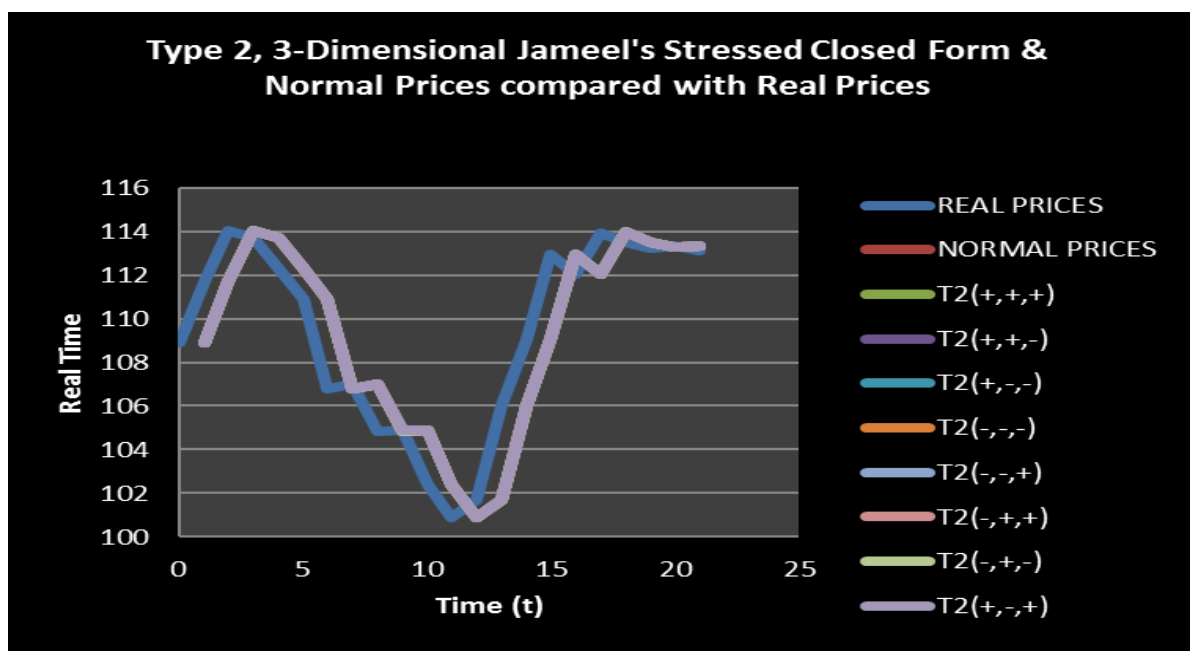

Figure 8. 


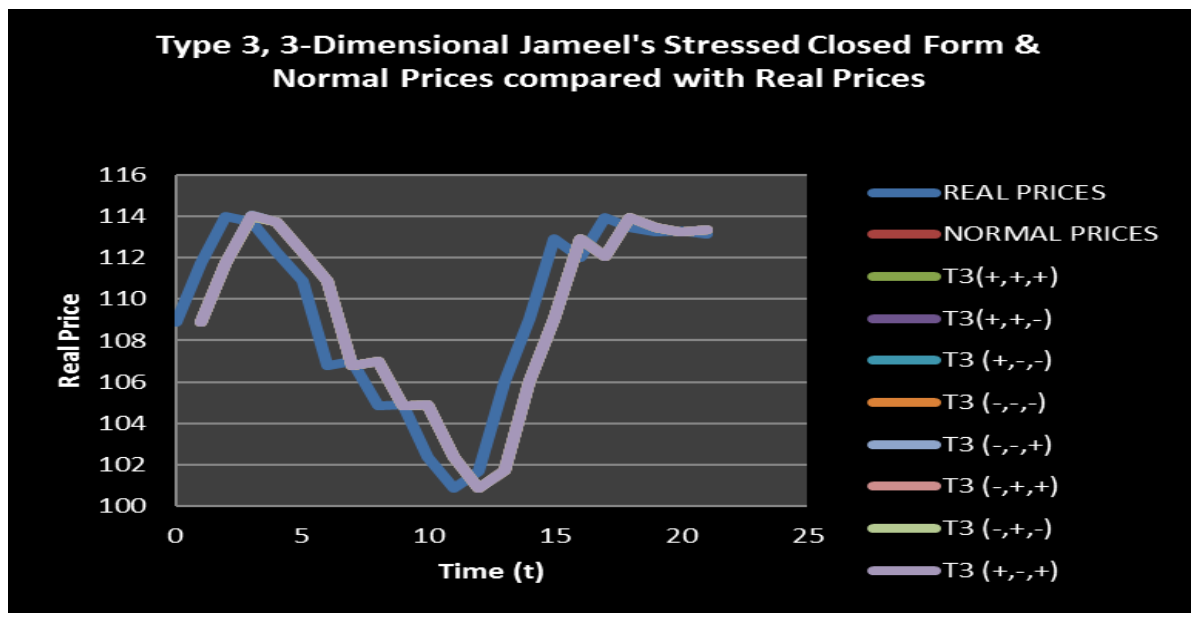

Figure 9.

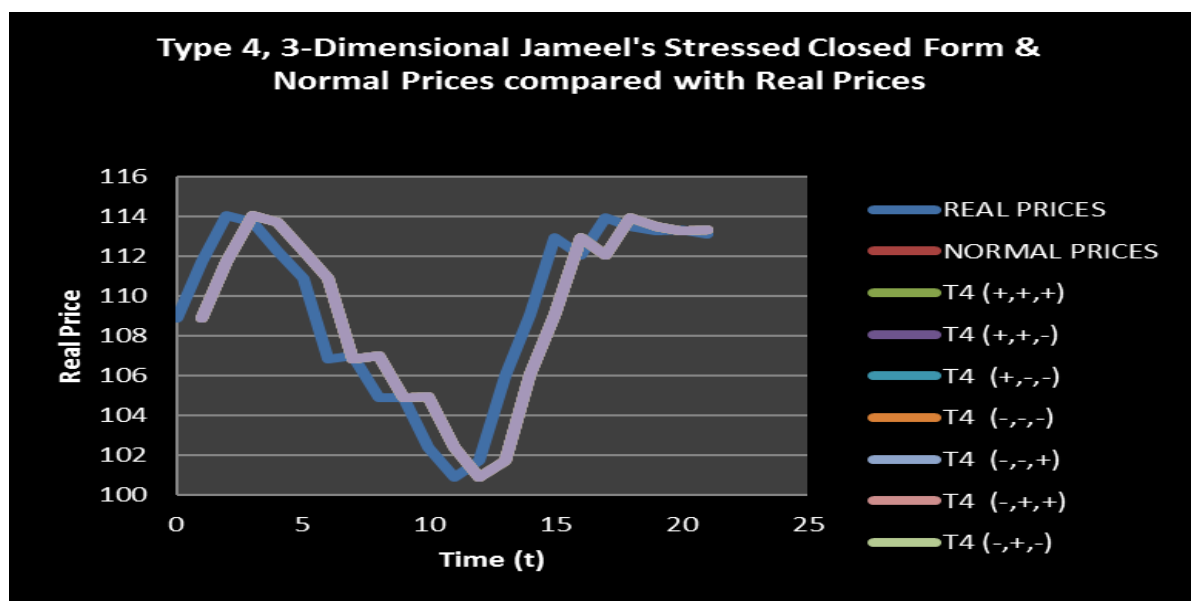

Figure 10.

It can be observed all the Shaded Areas in Table 1 to 8 approximated or almost coincided with the Chevron Corporation REAL PRICES. While figure 3 to 10 shows the performances of the proposed TWO and THREEDIMENSIONAL STRESSED CLOSED FORM PRICES vis-à-vis REAL PRICES and NORMAL PRICES.

More so, in the case of TWO-DIMENSIONAL, the four (4) proposed models ALTERNATES between ONLY TWO (2) VALUES at each point in time, similarly, in the case of THREE-DIMENSIONAL, the eight (8) proposed models also ALTERNATES between ONLY TWO (2) VALUES at each point in time.

The results performances were FASCINATINGLY interesting, impressive, viable, reliable, sophisticated and complaint with IFRS 9 since they incorporated the forward-looking information: (a) $\left\{\left(\mu_{A} \pm \sigma_{A} W_{J B 1}(t) \pm\right.\right.$ $\left.\left.\sigma_{A} W_{J B 2}(t)\right)\right\}, \mu_{A}$ is POSITIVE INFINITESIMAL, $\sigma_{A} \geq 1, W_{J B 1}(t)$ is a LOG-LOGISTIC $(3 \mathrm{P})$ and $W_{J B 2}(t)$ is a CAUCHY satisfying Jameel's Criterion for TWO-MENTIONAL; (b) $\left\{\left(\mu_{A} \pm \sigma_{A} W_{J B 1}(t) \pm \sigma_{A} W_{J B 2}(t) \pm\right.\right.$ $\left.\left.\sigma_{A} W_{J B 3}(t)\right)\right\}, \mu_{A}$ is POSITIVE INFINITESIMAL, $\sigma_{A} \geq 1, W_{J B 1}(t)$ is a LOG-LOGISTIC $(3 \mathrm{P}), W_{J B 2}(t)$ is a CAUCHY and $W_{J B 3}(t)$ is a BURR (4P) satisfying Jameel's Criterion for THREE-DIMENSIONAL and Geometric average of only positive Economic forecasts of the future Macroeconomic scenarios $\left\{\left(\mu_{A}\right)\right.$ and $\left.\left(\sigma_{A}\right)\right\}$ thereby minimizing the differences between Market Prices and Model Prices of the Financial 
Instruments.

Propose Jameel's Assets Approximation Theorem (JAAT)

Let $\left\{W_{J B 1}(t), W_{J B 2}(t), W_{J B 3}(t), \ldots, W_{J B n}(t): t \geq 0\right\}$ be a set of Non-Normal Fat-tailed Probability Distributions satisfies Jameel's Criterion with RANKING $1^{s t}, 2^{\text {nd }}, 3^{\text {rd }}, \ldots, n$th respectively. Let $\sigma_{A}$ be a Geometric Volatility of only positive Arithmetic Means of the Underlying Asset Return and Returns of the future economic forecasts of macroeconomic parameters and $\mu_{A}$ be a Geometric Means of only positive Arithmetic Means of the Underlying Asset Return and Returns of the future economic forecasts of macroeconomic parameters such that:

$L_{1}(t):=\mu_{A} \pm \sigma_{A} W_{J B 1}(t) \pm \sigma_{A} W_{J B 2}(t)$

$L_{2}(t):=\mu_{A} \pm \sigma_{A} W_{J B 1}(t) \pm \sigma_{A} W_{J B 2}(t) \pm \sigma_{A} W_{J B 3}(t)$

$L_{3}(t):=\mu_{A} \pm \sigma_{A} W_{J B 1}(t) \pm \sigma_{A} W_{J B 2}(t) \pm \sigma_{A} W_{J B(n-10)}(t)$

$L_{4}(t):=\mu_{A} \pm \sigma_{A} W_{J B 1}(t) \pm \sigma_{A} W_{J B 2}(t) \pm \sigma_{A} W_{J B(n-5)}(t) \pm \sigma_{A} W_{J B(n-4)}(t)$

.

$L_{n}(t):=\mu_{A} \pm \sigma_{A} W_{J B 1}(t) \pm \sigma_{A} W_{J B 2}(t) \pm \cdots \pm \sigma_{A} W_{J B n}(t)$

Then we generated a set of LINEAR COMBINATIONS $\left\{L_{1}(t), L_{2}(t), L_{3}(t), \ldots, L_{n}(t)\right\}$ of DIFFERENT DIMENSIONS, then $\exists L_{i}(t), i=1,2, \ldots, n$ such that the general form of JAMEEL'S SUBSTITUTIONS, TYPE 1 :

$\left(S_{J B}(t)\right)_{\text {Stressed }}=p_{0} \exp \left(\mu t+\sigma L_{i}(t)\right), i=1,2, \ldots, n$, will countably coincides at many points with the Asset's REAL PRICES or the difference between the MODEL PRICES and REAL PRICES will be very NEGLIGIBLE or even possibly ZERO at many points in time $t$. Note that one can work out for the other Three (3) TYPES.

\section{Discussion}

The Author set the Log-Logistic (3P) parameter $\xi$ to be 1 and Burr (4P) parameters $a=1, k=1, \gamma=1, \beta=$ 1 and $\alpha=2$ thus collapsed to almost Normal. With HIGH VALUES of $\xi, a, k, \gamma, \beta$, and $\alpha$, the proposed TWO and THREE DIMENSIONAL Jameel's Stressed Closed Prices TYPES will effectively approximates the REAL PRICES or the difference between the MODEL PRICES and REAL PRICES will be very NEGLIGIBLE or even possibly ZERO at many points in time $t$ as according to Proposed Jameel's Assets Approximation Theorem $(J A A T)$.

More so, if we could be able to Runs the Goodness of Fit Tests using Jameel's Criterion axiom known such as the RANKS of Kolmogorov Smirnov (KS) Test, Anderson-Darling Test, Jarque and Bera (JB) Test, Shapiro Wilk (SW) Test, Cramer-Von Mises Test, Pearson $\left(\chi^{2}\right.$ Godness of Fit $)$ Test, Lilliefors Corrected K-S Test, D'AgostinoSkewness Test, Anscombe-Glynn Kurtosis Test, D'Agostino-Pearson Omnibus are all UNITY (1) of the underlying Stock Returns then the proposed TWO and THREE DIMENSIONAL Jameel's Stressed Closed Prices TYPES will coincide at finitely many points with the REAL PRICES.

Also, as according to the study conducted by Jamilu (2015) where he considered Eleven (11) out of Fifty (50) World's Biggest Public Companies by FORBES as of 2015 Ranking regardless of the platform in which they are listed, Number of the Research Companies, Time Series (Short or Long), Old or Recently listed Companies using the time series from 2014 - 2009 with the aim of finding the Best Fitted Fat - Tailed Stocks Probability Distributions using Jameel's Criterion. Jamilu (2015) using Jameel's Criterion obtained Log-logistic (3P) as the First, Cauchy as Second and Burr (4P) as the Fourth, however, with ever changing of Information and Communication Technology, Natural Disasters, Terrorism, Political and Economic Risks, Strikes and other latent Risk Factors, one may uses Jameel's Criterion to obtain different Sets of NON-NORMAL, FAT-TAILED PROBABILITY DISTRIBUTIONS RANKING according to Jameel's Criterion to calculate different stressed closed form prices of different DIMENSIONS for IFRS 9 Compliance.

Furthermore, that we could TEST $\mu_{A}$ as ARITHMETIC Means of only positive Arithmetic Means of the Underlying Asset Return and Returns of the future economic forecasts of macroeconomic parameters, otherwise should remains GEOMETRIC MEANS as defined and used in the paper.

\section{Conclusion}

The paper uses Jameel's Criterion and Jameel's Contractional-Expansional Stress Methods to REPLACES the WEINER PROCESS $\{W(t)\}_{t \geq 0}$ with JAMEEL'S SUBSTITUTIONS FOR IFRS 9 COMPLIANCE with the 
forward-looking information: (a) $\left\{\left(\mu_{A} \pm \sigma_{A} W_{J B 1}(t) \pm \sigma_{A} W_{J B 2}(t)\right)\right\}, \mu_{A}$ is POSITIVE INFINITESIMAL, $\sigma_{A} \geq 1, W_{J B 1}(t)$ is a LOG-LOGISTIC (3P) and $W_{J B 2}(t)$ is a CAUCHY; (b) $\left\{\left(\mu_{A} \pm \sigma_{A} W_{J B 1}(t) \pm\right.\right.$ $\left.\left.\sigma_{A} W_{J B 2}(t) \pm \sigma_{A} W_{J B 3}(t)\right)\right\}, \mu_{A}$ is POSITIVE INFINITESIMAL, $\sigma_{A} \geq 1, W_{J B 1}(t)$ is a LOG-LOGISTIC (3P), $W_{J B 2}(t)$ is a CAUCHY and $W_{J B 3}(t)$ is a BURR (4P), where $W_{J B 1}(t), W_{J B 2}(t)$ and $W_{J B 3}(t)$ satisfies Jameel's Criterion.

The paper uses Jameels Substitutions: (i) $\left(\mu_{A} \pm \sigma_{A} W_{J B 1}(t) \pm \sigma_{A} W_{J B 2}(t)\right)$, whenever $\sigma_{A}>1, \mu_{A}$ is positive infinitesimal; (ii) $\left(\mu_{A} \pm W_{J B 1}(t) \pm W_{J B 2}(t)\right)$, whenever $\sigma_{A}=1, \mu_{A}$ is positive infinitesimal; (iii) $\left( \pm \sigma_{A} W_{J B 1}(t) \pm \sigma_{A} W_{J B 2}(t)\right)$, whenever $\sigma_{A}>1, \mu_{A}=0$; and (iv) $\left( \pm W_{J B 1}(t) \pm W_{J B 2}(t)\right)$, whenever $\sigma_{A}=1$, $\mu_{A}=0$ for TWO-DIMENASIONAL proposed stressed closed form solutions and (1) $\left(\mu_{A} \pm \sigma_{A} W_{J B 1}(t) \pm\right.$ $\left.\sigma_{A} W_{J B 2}(t) \pm \sigma_{A} W_{J B 3}(t)\right)$, whenever $\sigma_{A}>1, \mu_{A}$ is positive infinitesimal; (2) $\left(\mu_{A} \pm W_{J B 1}(t) \pm W_{J B 2}(t) \pm\right.$ $\left.W_{J B 3}(t)\right)$, whenever $\sigma_{A}=1, \mu_{A}$ is positive infinitesimal; (3) $\left( \pm \sigma_{A} W_{J B 1}(t) \pm \sigma_{A} W_{J B 2}(t) \pm \sigma_{A} W_{J B 3}(t)\right)$, whenever $\sigma_{A}>1, \mu_{A}=0$; and (4) $\left( \pm W_{J B 1}(t) \pm W_{J B 2}(t) \pm W_{J B 3}(t)\right)$, whenever $\sigma_{A}=1, \mu_{A}=0$ for THREE-DIMENSIONAL proposed stressed closed form solutions thereby coming up with Eight (8) proposed stressed closed form solutions for IFRS 9 Compliance.

The paper tested the performances of the Eight (8) proposed stressed closed form solutions with the aid of Chevron Corporation (CVX) Stock data extracted from yahoo finance, time series from 2014 - 1991. The results were fascinatingly interesting, impressive, viable and reliable, sophisticated, and complaint with IFRS 9 since they incorporated MORE forward-looking information and Economic forecasts of the future macroeconomic parameters thereby minimizing the differences between market prices and models prices.

Finally, the results performances of the PROPOSED TWO and THREE DIMENSIONAL STRESSED CLOSED FORM SOLUTIONS of Ornstein - Uhlenbeck Process, Cox-Ingersoll-Ross (1985) Model, Vasicek Model, BlackKarasinki (1991) Model, Chen (1994) Model, Kalotay - Williams - Fabozzi (1993) Model, Longstaff - Schwatz (1992) Model, Ho-Lee Model (1986) Model, Hull-White (1990) Model, Black-Derman-Toy (1990) Model, Heston Volatility Model and ETFs and Leveraged ETFs Models can be TESTED using the processes as in the case of TWO and THREE DIMENTIONAL stressed closed form solution of the GEOMETRIC BROWNIAN MOTION presented in this paper. Also, the models would provide excellent results using MONTE-CARLO or GENERAL SIMULATION ANALYSES.

\section{References}

Adegoke, O. (1979). Introduction to Real Analysis, Department of Mathematics, University of Ibadan, Girardet Press (W.A) Co; Ibadan.

Andrew, W. L., \& GraigMackinlay, A. (1999). A Non-Random Walk Down Wall Street, Princeton University Press, 41 William Street Princeton, New Jersey, 08540, United Kingdom.

Arditti, F. D. (1996). Derivatives: A Comprehensive Resource for Options, Futures, Interest Rate Swaps, and Mortgage Securities. Boston: Harvard Business School Press. ISBN0-87584560-6.

Barnaby, B., et al. (2016). Complying with IFRS 9 Impairment Calculations for Retail Portfolios, Moody's Analytics Risk Perspectives, the convergence of Risk, Finance, and Accounting, Volume VII, June, 2016. 
Bellotti, T., \& Crook, J. (2012). Loss Given Default Models Incorporating Macroeconomic Variables for Credit Cards. International Journal of Forecasting, 28(1), 171-182. https://doi.org/10.1016/j.ijforecast.2010.08.005

Bhatt, B. J., et al. (2013). Fractional Brownian Motion and Predictability Index in Financial Market. Global Journal of Mathematical Sciences: Theory and Practical, 5(3), 97-203.

Bialkowska, E., \& Chai, A. (2017). Banks-IFRS 9 Pre-transition Disclosure: Guide to Annual Financial Statements for Banks, KPMG, IFRG Limited, 1-18.

Borio, C., \& Lowe, P. (2001). To Provision or not to Provision. BIS Quarterly Review, 9(3), 36-48.

Burton, G. M. (1999). A Random Walk Down Wall Street, W.W. Norton \& Company, Inc. 500 fifth Avenue, New York, N. Y. 10110.

Burton, G. M. (2009). The Clustering of Extreme Movements: Stock prices and the Weather, Princeton University, AtanuSaha, Alixpartners, Alex Grecu, Huron Consulting Group, CEPS working paper No. 186 February, 2009.

Daniel, P. (2006). Estimating Probabilities of Default for German Savings Banks and Credit Cooperatives, University of Applied Sciences, Mainz, Ander Bruchspitze, 50, D - 55122 Mainz.

David, M. R. (2012). Simulating Default Probabilities in Stress Scenarios, Presented to the PRMIA Global Risk Conference, New York, NY, May 14, 2012.

David, N. (2006). Fractional Brownian Motion: Stochastic Calculus and Applications; Proceedings of the International Congress of Mathematicians, Madrid, Spain, 2006, European Mathematical Society

Dieker, T. (2004). Simulation of Fractional Brownian Motion, Thesis, University of Twente, Department of Mathematical Sciences, P.O. BOX 217, 7500 AE Enschede, Netherlands.

Jamilu, A. A. (2013). A Guide to Financial Mathematics and Risk Management for Nigeria, Book Published by Delcon Press, Suleja, Niger, ISBN: 978-223-529-6, First Edition 2013.

Jamilu, A. A. (2014). Modern Approach to Financial Risk Management, Book Published by Delcon Press, Suleja, Niger, ISBN: 978-978-942-265-4, First Edition 2014.

Jamilu, A. A. (2014). Understanding Financial Risks, Book Published by Delcon Press, Suleja, Niger, ISBN: ISBN: 978-978-942-266-1, First Edition 2014.

Jamilu, A. A. (2015). Banking and Economic Advanced Stressed Probability of Default Models, Accepted for Publication in the Asian Journal of Management Sciences, AJMS/2015/ May - June - 303 - 798 - 2 - RV.

Jamilu, A. A. (2015). Estimation of Probability of Default using Advanced Stressed Probability of Default Models, Ongoing Ph.D Thesis, Ahmadu Bello University (ABU), Zaria, Nigeria.

Jamilu, A. A. (2018). IFRS 9 Measurement of Financial Instruments 2018: Jameel's Non-Normal Brownian Motion Models are Indeed IFRS 9 Complaint Models, accepted paper in the Journal of Economics and Management Sciences.

Krishna, R., \& Vaughan, C. (2016). Simulating Stock Prices using Geometric Brownian Motion: Evidence from Australian Companies, Australasian Accounting. Business and Finance Journal, 10(3), 23-47. https://doi.org/10.14453/aabfj.v10i3.3

Nassim, N. T. (2007). Black Swans and the Domains of Statistics. American Statistician, August 2007, 6(3).

Nassim, N. T. (2009). Errors, Robustness, and Fourth Quadrant, New York University Polytechnic Institute and Universa Investment, United States. International Journal of Forecasting, 25(2009), 744-759. https://doi.org/10.1016/j.ijforecast.2009.05.027

Nassim, N. T. (2010). Convexity, Robustness, and Model Error inside the "Black Swan Domain”, Draft Version, September, 2010.

Nassim, N. T. (2010). Why Did the Crisis of 2008 Happen, Draft, $3^{\text {rd }}$ Version, August, 2010.

Nassim, N. T. (2011). A Map and Simple Heuristic to Detect Fragility, Antifragility, and Model Error, First Version, 2011.

Nassim, N. T. (2011). The Future has Thicker Tails Past: Model Error as Branching Counterfactuals, presented in Honor of Benoit Mandelbrot's at his Scientific Memorial, Yale University, April, 2011.

Nassim, N. T. (2012). The Illusion of Thin - Tails under Aggregation, NYU - Poly, January, 2012.

Nassim, N. T., et al. (2009). Risk Externalities and Too bid to Fail, New York University Polytechnic Institute, 
11201, New York, United States.

Onali, E., \& Ginesti, G. (2014). Pre-adoption Market Reaction to IFRS 9: A Cross-country Event-study. Journal of Accounting and Public Policy, 33(6), 628-637. https://doi.org/10.1016/j.jaccpubpol.2014.08.004

Reney, D. E., \& Michael, R. M. (2016). Forecasting of Stock Prices using Brownian Motion - Monte Carlo Simulation, Proceedings of the 2016 International Conference on Industrial Engineering and Operations Management, Kuala Lumpur, Malaysia, March 8-10, 2016.

Rogers, L. C. G. (1997). Arbitrage with Fractional Brownian Motion, Mathematical Finance, 7(1), (January, 1997), 95-105. https://doi.org/10.1111/1467-9965.00025

Spreedhar, T. B., et al. (2004). Forecasting Default with the KMV - Merton Model, University of Michigan, Ann Arbor MI 48109.

Steven, R. D. (2013). Stochastic Processes and Advanced Mathematical Finance, The Definition of Brownian Motion and the Wiener process, Department of Mathematics, 203 Avery Hall, University of Nebraska-Lincoln, Lincoln, NE 68588-0130.

Tidaruk, A. (2014). Mathematical Model of Stock Prices via a Fractional Brownian Motion Model with Adaptive Parameters.

Van Ness (1968). Fractional Brownian Motions, Fractional Noises and Applications (M \& Van Ness (1968)), SIAM Review: 10, 1968, 422-437. https://doi.org/10.1137/1010093

Wang, B., et al. (2012). When Brownian Diffusion is no Gaussian. Nature Materials, 11(June, 2012), Macmillian Publishers Limited. https://doi.org/10.1038/nmat3308

Zhang, W. Y. (2015). Introduction to Ito's Lemma, Lecture Note, Cornell University, Department of Statistical Sciences, May 6, 2015.

\section{Copyrights}

Copyright for this article is retained by the author(s), with first publication rights granted to the journal.

This is an open-access article distributed under the terms and conditions of the Creative Commons Attribution license (http://creativecommons.org/licenses/by/4.0/). 